\title{
Acoustic anisotropy in uniaxial tungsten bronze ferroelectric single crystals studied by Brillouin light scattering
}

\author{
Jae-Hyeon Ko, ${ }^{1, a)}$ S. G. Lushnikov, ${ }^{2}$ Do Han Kim, ${ }^{3}$ Seiji Kojima, ${ }^{3}$ Byeong-Eog Jun, ${ }^{4}$ and \\ Yoon Hwae Hwang ${ }^{5}$ \\ ${ }^{1}$ Department of Physics, Hallym University, 39 Hallymdaehakgil, Chuncheon, Gangwondo 200-702, \\ Republic of Korea \\ ${ }^{2}$ A. F. Ioffe Physical Technical Institute, Russian Academy of Sciences, St. Petersburg 194021, Russia \\ ${ }^{3}$ Institute of Materials Science, University of Tsukuba, Tsukuba City, Ibaraki 305-8573, Japan \\ ${ }^{4}$ Department of Physics and Basic Science Research Institute, Pukyong National University, Busan 608-737, \\ Republic of Korea \\ ${ }^{5}$ Department of Nanomaterials, Pusan National University, Miryang 627-706, Republic of Korea
}

(Received 2 July 2008; accepted 1 October 2008; published online 18 November 2008)

\begin{abstract}
Acoustic properties were investigated for four tungsten bronze (TB) uniaxial ferroelectric crystals, i.e., $\left(\mathrm{Sr}_{0.61} \mathrm{Ba}_{0.39}\right)_{5} \mathrm{Nb}_{10} \mathrm{O}_{30}(\mathrm{SBN61})$, Cu-doped $\left(\mathrm{K}_{0.5} \mathrm{Na}_{0.5}\right)_{1.0}\left(\mathrm{Sr}_{0.75} \mathrm{Ba}_{0.25}\right)_{4.5} \mathrm{Nb}_{10} \mathrm{O}_{30}(\mathrm{KNSBN}: \mathrm{Cu})$, $\mathrm{K}_{5.80} \mathrm{Li}_{3.82} \mathrm{Nb}_{10.12} \mathrm{O}_{30}$, and $\mathrm{K}_{4.74} \mathrm{Li}_{3.07} \mathrm{Nb}_{10.44} \mathrm{O}_{30}$ of which the spontaneous polarization is directed along the polar $c$ axis. Large acoustic anisotropy between the two elastic constants $C_{11}$ and $C_{33}$ have been observed from all samples. $C_{33}$ exhibits a significant softening on approaching the diffuse phase transition temperature from high-temperature side while $C_{11}$ does not show any substantial change in the same temperature range. This softening is accompanied by substantial growth of hypersonic damping, appearance and growth of central peak $(\mathrm{CP})$, and slowing down of the relevant dynamics of $\mathrm{CP}$ represented by the reducing half width. All these results indicate that the lattice motions along the $c$ axis couple strongly to the one-component order parameter of the polar nanoregions (PNRs) or precursor polar clusters which form and grow below a certain temperature in the paraelectric phase. The inverse dielectric constant measured along the $c$ axis of SBN61 and KNSBN:Cu can be described by two linear regions divided by a crossover temperature at which the change in the magnitude of dipole moments and the strength of the dipole couplings are expected due to the formation of PNRs. $C_{33}$ of SBN61 becomes continuously softened upon cooling even when the temperature crosses the Burns temperature $T_{B}$ at which PNRs begin to appear. It may suggest that additional relaxation process other than that of PNRs may exist at high temperatures above $T_{B}$ and couple to the longitudinal acoustic waves propagating along the polar axis. Recent observation of a single-particle relaxation at high temperatures and its transformation into a collective relaxation of PNRs by dielectric spectroscopy [Belous et al., J. Appl. Phys. 102, 014111 (2007)] might be related to the anomalous acoustic behavior of SBN61 observed above $T_{B}$. C 2008 American Institute of Physics. [DOI: 10.1063/1.3021107]
\end{abstract}

\section{INTRODUCTION}

Relaxor ferroelectrics (RFEs) have been an intense research subject for a few decades owing to the high applicability in various fields as well as their complex structural and dynamical properties. ${ }^{1-3}$ RFEs exhibit several common features such as a diffuse frequency-dependent dielectric permittivity of which the maximum temperature $T_{m}$ follows the Vogel-Fulcher relationship, ${ }^{4}$ the absence of structural phase transition, ${ }^{5}$ and a formation of polar nanoregions (PNRs) at a certain temperature, the so-called Burns temperature $\left(T_{B}\right)$, which is normally higher than $T_{m}$ by a few hundred degrees. ${ }^{6}$ Among them, the exact nature of PNRs has been considered as the central problem in the fundamental understanding of RFEs since PNRs are believed to be responsible for the complex dynamics and many unique properties of RFEs. ${ }^{7,8}$ The existence of $T_{B}$ at which PNRs begin to appear is correlated with many unusual properties of RFEs such as the deviation

${ }^{a)}$ Electronic mail: hwangko@hallym.ac.kr. of the dielectric constant from the high-temperature CurieWeiss law, ${ }^{4}$ appearance of diffuse elastic scattering from $\mathrm{x}$-ray and neutron scatterings, ${ }^{9,10}$ deviations of the index of refraction and the volume of the cubic cell from hightemperature linear behaviors, ${ }^{6,11,12}$ occurrence of acoustic emission signals, ${ }^{13}$ etc. However, $T_{B}$ should not be considered as a usual phase transition temperature since there is no structural change in the crystal on a macroscopic or mesoscopic scale.

The microscopic origin of the formation of PNRs is not fully understood at the moment. The lattice disorder introduced into the perovskite structure by chemical substitution or lattice defects has been suggested to be one of the key ingredients for the formation of PNRs. ${ }^{8}$ Recently, it was suggested from first-principle-based simulations ${ }^{14}$ that PNRs are predominantly pinned to the quenched chemically ordered regions (CORs), which are formed by the two different cations with dissimilar valence states occupying the perovskite $B$ sites. Due to the different valance states, these short-range CORs may induce charge disorder and thus become the 
TABLE I. The composition of the investigated TB crystals, their maximum temperatures $\left(T_{m}\right)$, the real part of the complex dielectric constant at $T_{m}$ measured along the $c$ axis $\left(\epsilon_{c}^{\prime}\right)$, and crossover temperatures $\left(T_{\text {cross }}\right)$.

\begin{tabular}{lcccc}
\hline \hline Crystal & Composition & $T_{m}(2 \mathrm{kHz})$ & $\epsilon_{c}^{\prime}(2 \mathrm{kHz})$ & $T_{\text {cross }}$ \\
\hline SBN61 & $\left(\mathrm{Sr}_{0.61} \mathrm{Ba}_{0.39}\right)_{5} \mathrm{Nb}_{10} \mathrm{O}_{30}$ & $\sim 341 \mathrm{~K}$ & 50900 & $630-680 \mathrm{~K}$ \\
KNSBN:Cu & $\left(\mathrm{K}_{0.5} \mathrm{Na}_{0.5}\right)_{1.0}\left(\mathrm{Sr}_{0.75} \mathrm{Ba}_{0.25}\right)_{4.5} \mathrm{Nb}_{10} \mathrm{O}_{30}$ & $\sim 420 \mathrm{~K}$ & 19200 & $\sim 530 \mathrm{~K}$ \\
$\mathrm{KLN}-\mathrm{a}$ & $\mathrm{K}_{5.80} \mathrm{Li}_{3.82} \mathrm{Nb}_{10.12} \mathrm{O}_{30}$ & $\sim 725 \mathrm{~K}$ & $2150^{\mathrm{b}}$ & $\cdots$ \\
KLN-b & $\mathrm{K}_{4.74} \mathrm{Li}_{3.07} \mathrm{Nb}_{10.44} \mathrm{O}_{30}$ & $\sim 705 \mathrm{~K}$ & $1140^{\mathrm{b}}$ & $\cdots$ \\
\hline \hline
\end{tabular}

${ }^{\mathrm{a}}$ Doped with 0.04 wt $\%$ of $\mathrm{CuO}$.

${ }^{\mathrm{b}}$ Measured at $10 \mathrm{kHz}$.

source of quenched random fields in RFEs. However, clear relationship between PNRs and CORs is still far from clear understanding, and more thorough experimental and theoretical efforts are necessary in order to reveal whether the compositional heterogeneity is prerequisite to the formation of PNRs. ${ }^{15}$ In the case of tungsten bronze (TB) uniaxial relaxor crystals such as strontium barium niobate $\left[\mathrm{Sr}_{1-x} \mathrm{Ba}_{x} \mathrm{Nb}_{2} \mathrm{O}_{6}\right.$ $(\mathrm{SBN})]$, quenched random fields are expected to arise from random empty positions on the $A$-sites of unfilled structure. ${ }^{16}$ Depending on the cation ions and their site occupancies, TB crystals show various interesting phase sequences, among which the ferroelectric phase transition from hightemperature tetragonal $4 / \mathrm{mmm}$ to low-temperature tetragonal $4 \mathrm{~mm}$ symmetry has been the most extensively studied. In contrast to the quasicontinuous nature of the order parameter of cubic perovskite RFEs due to the multiple easy directions in the cubic primitive cell, ${ }^{17}$ the direction of the order parameter of the tetragonal uniaxial TB RFEs such as SBN is directed along the fourfold polar axis, which can be modeled by the one-component Ising spins. ${ }^{18}$

Due to the random directions of polarizations of PNRs, macroscopic polarization cannot be observed in the ergodic relaxor phase above $T_{m}$ under the zero-field-cooling condition. However, since the square of the local polarizations of PNRs is not zero, several physical properties coupled to PNRs via electrostriction may show abnormal behaviors when PNRs begin to appear at $T_{B}$. The appearance of PNRs can also be noticed from changes in acoustic properties, i.e., the sound velocity and the corresponding elastic constant at $T_{B} \cdot{ }^{19}$ High-temperature acoustic anomalies near $T_{B}$ of many lead-based perovskite relaxors have been investigated by Brillouin light scattering technique. ${ }^{20-26}$ The Brillouin frequency shift $\left(\nu_{B}\right)$ of the longitudinal acoustic (LA) waves, corresponding to the elastic constant $C_{11}$, first shows a typical hardening and then exhibits a substantial softening on approaching $T_{m}$ upon cooling starting from high temperatures above $T_{B}$. This softening has been accompanied by a significant increase in the hypersonic damping as well as the appearance and growth of the central peak (CP), indicating that the dynamics of PNRs is strongly coupled to the acoustic waves in RFEs. From these acoustic anomalies, important information about the dynamics of PNRs could be derived such as the temperature dependence of the relaxation time and its distribution, acoustic dispersion, etc. In contrast to these detailed information on the acoustic properties of cubic perovskite RFEs, there has been no report on the acoustic properties of uniaxial RFEs in a high-temperature range covering $T_{B}$. The temperature dependence of several elastic con- stants of $\mathrm{Sr}_{0.61} \mathrm{Ba}_{0.39} \mathrm{Nb}_{2} \mathrm{O}_{6}$ (SBN61) has been studied by Brillouin scattering in a limited temperature range. ${ }^{27}$ It was shown that the LA mode propagating along the polar $c$ axis and the corresponding elastic constant $C_{33}$ exhibit a substantial softening on approaching the diffuse ferroelectric phase transition temperature accompanied by a significant increase in the hypersonic damping as well as in the integrated intensity of $\mathrm{CP}$. The detailed temperature dependence of the $\mathrm{CP}$ has recently been studied by broadband Brillouin scattering and revealed that $\mathrm{CP}$ has two components among which the $\mathrm{CP}$ component in a narrower frequency range appears due to the formation and growth of PNRs starting from $T_{B}{ }^{28}$ Preliminary Brillouin scattering study on $\left(\mathrm{K}_{0.5} \mathrm{Na}_{0.5}\right)_{1.0}\left(\mathrm{Sr}_{0.75} \mathrm{Ba}_{0.25}\right)_{4.5} \mathrm{Nb}_{10} \mathrm{O}_{30}$ doped with 0.04 wt \% of $\mathrm{CuO}(\mathrm{KNSBN}: \mathrm{Cu})$ has been reported by Siny et al. ${ }^{29} \nu_{B}$ corresponding to $C_{33}$ of this crystal shows a steplike anomaly at the phase transition temperature and has been ascribed to the electrostrictive coupling between the order parameter and the strain. The purpose of the present study is to investigate the acoustic properties of SBN61 in a widest temperature range including $T_{B}$ and to find correlation between the formation of PNRs and the sound velocity propagating along the polar axis. For comparison, acoustic behaviors of three other TB ferroelectric single crystals, KNSBN:Cu, $\mathrm{K}_{5.80} \mathrm{Li}_{3.82} \mathrm{Nb}_{10.12} \mathrm{O}_{30}$ (KLN-a), and $\mathrm{K}_{4.74} \mathrm{Li}_{3.07} \mathrm{Nb}_{10.44} \mathrm{O}_{30}$ (KLN-b) have been investigated and compared. Special attention has been paid to the comparison between $C_{11}$ and $C_{33}$ since they are believed to represent the acoustic anisotropy inherent in these uniaxial TB single crystals.

\section{EXPERIMENT}

SBN61, KNSBN:Cu, and two KLN single crystals were grown by the Czochralski method, and the detailed sample growth conditions can be found elsewhere. ${ }^{30}$ The compositions of the four investigated TB crystals, their dielectric maximum temperatures $\left(T_{m}\right)$ evaluated at a certain probe frequency, the absolute values of the real part of the complex dielectric constant at $T_{m}$ measured along the $c$ axis $\left(\epsilon_{c}^{\prime}\right)$, and crossover temperatures which will be discussed in Secs. III and IV are listed in Table I. Platelet samples were cut along several crystallographic axes from single crystals and polished to optical quality. A Sandercock-type six-pass tandem Fabry-Pérot interferometer has been used to measure the Brillouin spectra of these single crystals. Either a backward scattering geometry or a special right-angle scattering geometry (called 90A scattering) (Ref. 31) was used for the measurement of the Brillouin spectrum. The phonon wavevector 


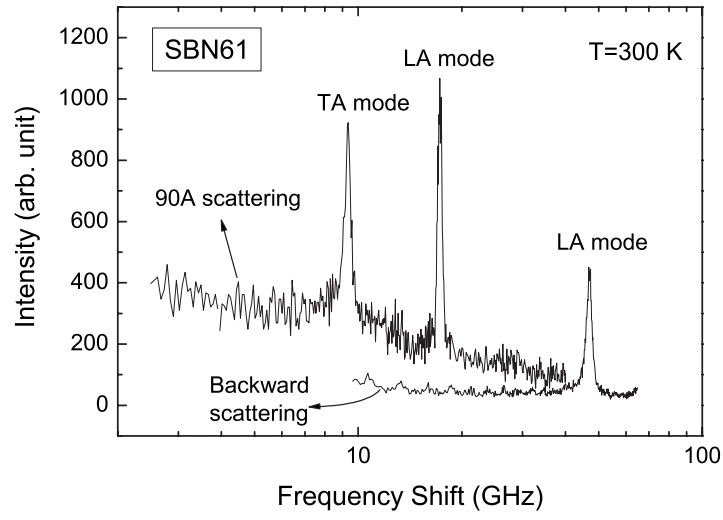

FIG. 1. Two Brillouin spectra of SBN61 at room temperature measured at the two scattering geometries where the wavevector of the probed phonons is parallel to the polar $c$ axis.

was set to be along either the polar $c$ axis or $a$ axis when the backscattered light was monitored in order to obtain $C_{33}$ or $C_{11}$. In the $90 \mathrm{~A}$ scattering geometry, the Brillouin spectrum was obtained at the $\bar{a}(c c) \bar{b}$ scattering geometry, where $\bar{a}$ and $\bar{b}$ denote the directions of the incident and scattered lights in the plane perpendicular to the $c$ axis. $\bar{a}$ and $\bar{b}$ were inclined by $45^{\circ}$ with respect to the tetragonal $a$ and $b$ axes, and the phonon propagation direction was along the tetragonal $a($ $=b)$ axis. In addition, the crystal plate was rotated by $90^{\circ}$ in the way by which the phonon propagation direction was set to be along the tetragonal $c$ axis. The former was used to probe $C_{11}$ and $\mathrm{CP}$ while the latter to probe $C_{33}$. Two free spectral ranges of 75 and $30 \mathrm{GHz}$ were used for the backward and 90A scattering geometries, respectively. The wavelength of the laser beam was either 532 or $488 \mathrm{~nm}$. All the Brillouin spectra were recorded during the cooling process.

\section{RESULTS}

Figure 1 shows two Brillouin spectra of SBN61 at room temperature measured at the two scattering geometries where the wavevector of the probed phonons is parallel to the polar $c$ axis. In this case, the corresponding elastic constant of the LA mode is $C_{33}$. The Brillouin frequency shift $\nu_{B}$ is determined by the sound velocity $V$ and the phonon wavevector $q$, which can be expressed by the following equation:

$$
\nu_{B}=\frac{q V}{2 \pi}=\frac{2 n \sin (\theta / 2)}{\lambda} V .
$$

In this expression, $n$ denotes the refractive index corresponding to the polarization of the incident light, $\theta$ the scattering angle, and $\lambda$ the wavelength of the incident laser beam. The magnitudes of the wavevector $q$ are $1.67 \times 10^{7}$ and 5.57 $\times 10^{7} \mathrm{~m}^{-1}$ for the $90 \mathrm{~A}$ scattering and backscattering geometries, respectively, from which the large difference in $\nu_{B}$ of the LA waves shown in Fig. 1 can be justified. The sound velocity of the LA mode can be calculated from the measured $\nu_{B}$ and the reported value of the refractive index. ${ }^{32}$ The measured spectrum was fitted by the Lorentzian function convoluted by the Gaussian instrumental function resulting in accurate values for the $\nu_{B}$ and the full width at half maxi-

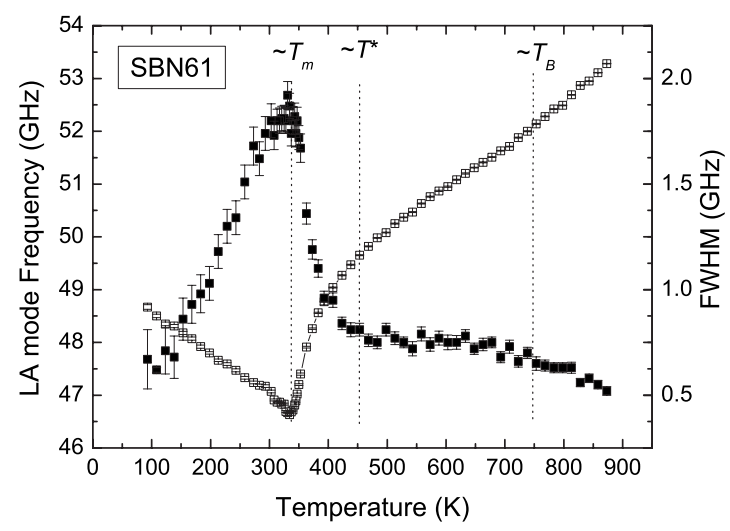

FIG. 2. The temperature dependence of the $\nu_{B}$ and the FWHM of the Brillouin doublet of SBN61 arising from the LA waves propagating along the polar $c$ axis measured at the backward scattering geometry.

mum (FWHM) of the Brillouin doublet arising from the acoustic waves.

Figure 2 displays the temperature dependence of the $\nu_{B}$ and the FWHM of the Brillouin doublet corresponding to the LA waves propagating along the polar $c$ axis measured at the backward scattering geometry. $\nu_{B}$ decreases continuously upon cooling from the highest measurement temperature of about $873 \mathrm{~K} . T_{B}$ of SBN61 is known to be about $750 \mathrm{~K}$ estimated from the linear birefringence study. ${ }^{12} \nu_{B}$ becomes smaller continuously with decreasing temperature even when crossing $T_{B}$. A slight change in the slope of $\nu_{B}$ seems to be noticeable near $T_{B}$. The softening of $\nu_{B}$ becomes more substantial below a certain temperature of $T^{*} \sim 450 \mathrm{~K}$ below which the integrated intensities of CPs increase ${ }^{28}$ and the FWHM of the LA mode in Fig. 2 also grows significantly. This temperature is similar to the one at which the intensity of the second harmonic generation signal suddenly increases upon cooling. ${ }^{33} \nu_{B}$ and the FWHM of the LA mode show a minimum and a maximum, respectively, at the temperature near $T_{m}$. Below $T_{m}, \nu_{B}$ hardens and the FWHM decreases with lowering temperature.

The Brillouin spectrum of SBN has also been measured at the $90 \mathrm{~A}$ scattering geometry. Two sample orientations have been used where the direction of the phonon wavevector is along either the tetragonal $c$ or $a$ axis in each geometry. The advantage of the present scattering geometry is that the sound velocity can be obtained without the knowledge of the refractive index of the single crystals. ${ }^{34}$ The sound velocity $V$ is related to the Brillouin frequency shift $\nu_{B}$ as

$$
V=\frac{\lambda_{0} \nu_{B}}{\sqrt{2}},
$$

where $\lambda_{0}$ is the laser wavelength in vacuum. The elastic stiffness coefficient $C_{i j}$ is related to the corresponding sound velocity $V$ and the density of the crystal $\rho$ according to the following equation:

$$
C_{i j}=\rho V^{2} .
$$

From Eq. (3), $C_{11}$ and $C_{33}$ have been obtained using the theoretically calculated density of SBN61 $\left(\rho=5.3 \mathrm{~g} / \mathrm{cm}^{3}\right)$ based on the reported lattice parameters, ${ }^{35}$ and the results are summarized in Fig. 3. The intensity of the Brillouin doublet 


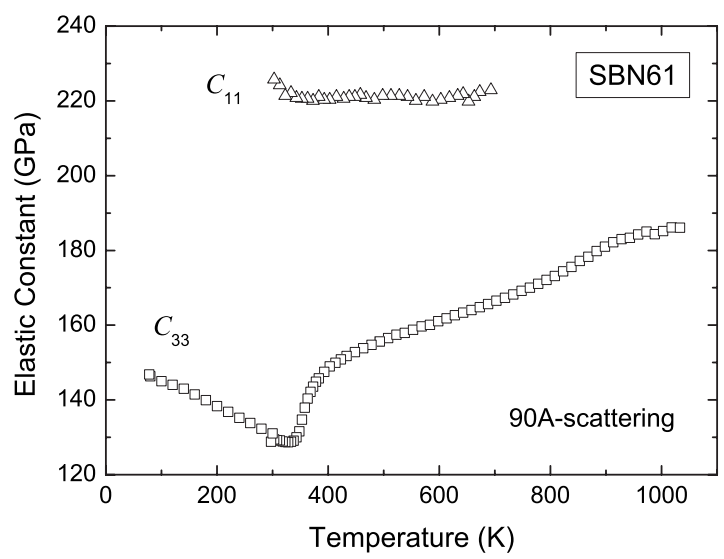

FIG. 3. The temperature dependence of $C_{11}$ and $C_{33}$ elastic constants of SBN61 measured by the 90A scattering geometry.

due to the TA mode becomes very weak near $300 \mathrm{~K}$, below which we could not obtain reliable data for $C_{11}$. Although the measurement temperature range for obtaining $C_{11}$ is rather limited, it is clear that the LA waves whose wavevector is in the plane perpendicular to the polar axis do not show any substantial change near the diffuse ferroelectric phase transition, in contrast to the LA waves propagating along the polar $c$ axis of which the corresponding elastic constant $C_{33}$ experiences a significant softening on approaching $T_{m}$.

$\nu_{B}$ 's of the LA waves of KNSBN:Cu propagating along the two directions parallel and perpendicular to the tetragonal $c$ axis are shown in Fig. 4(a). The FWHM of the LA mode whose phonon wavevector is parallel to the $c$ axis is displayed in Fig. 4(b). Similar to the case of SBN61, acoustic anisotropy is clearly seen in this TB uniaxial crystal. The change in $\nu_{B}$ of the LA waves due to the temperature change from 600 to $400 \mathrm{~K}$ is approximately 0.8 and $4 \mathrm{GHz}$ for the phonons propagating along the $a$ and $c$ axes, respectively.

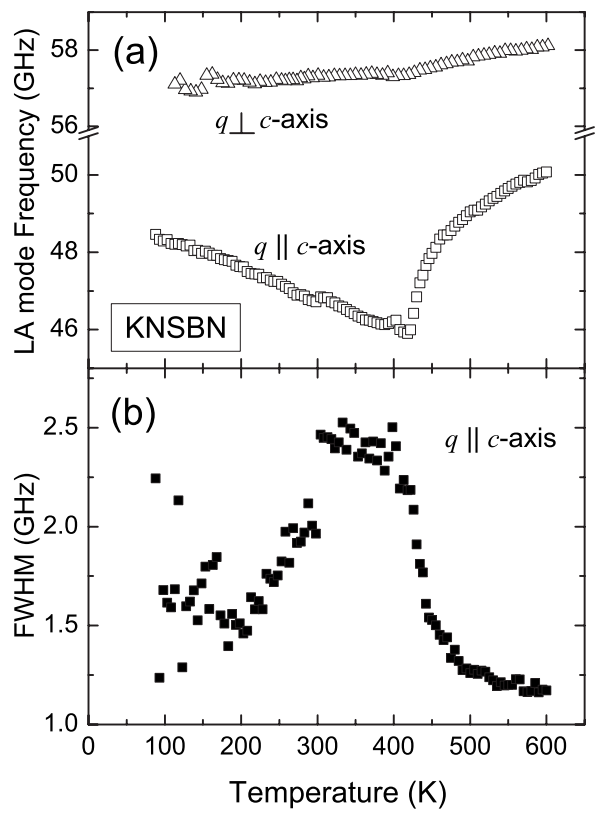

FIG. 4. (a) The temperature dependence of $\nu_{B}$ 's of the LA waves of KNS$\mathrm{BN}: \mathrm{Cu}$ propagating along the two directions parallel and perpendicular to the tetragonal $c$ axis. (b) The temperature dependence of the FWHM of the LA mode whose phonon wavevector is parallel to the $c$ axis.

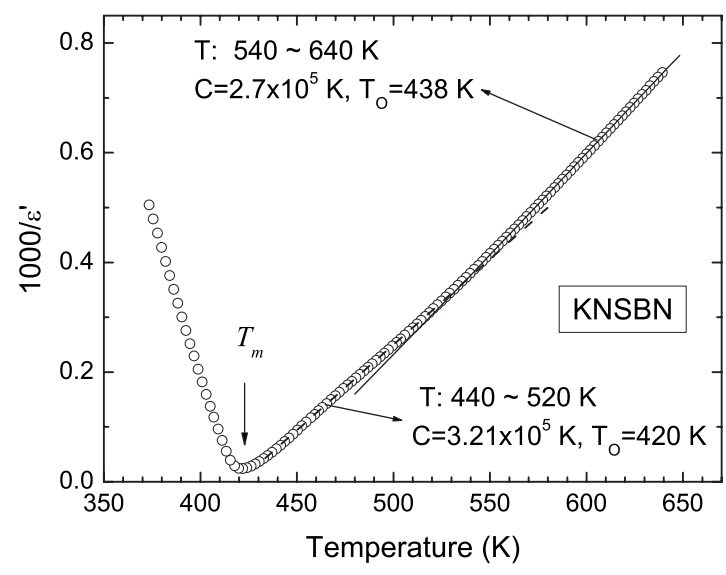

FIG. 5. The inverse dielectric constant of KNSBN:Cu measured long the $c$ axis and the fitted results denoted as two lines along with the fitting parameters and the temperature ranges used for the fitting procedure:

Regarding the change in the hypersonic damping, the FWHM begins to increase at about $530 \mathrm{~K}$ upon cooling. The complex dielectric constant of $\mathrm{KNSBN}: \mathrm{Cu}$ was reported in our previous study. ${ }^{36}$ The inverse of the real part of the dielectric constant, $1 / \epsilon^{\prime}$, measured along the polar $c$ axis shows two linear regions in the paraelectric phase, and the crossover temperature is approximately $530 \mathrm{~K}$. This is illustrated in Fig. 5 which shows two linear regions in $1 / \epsilon^{\prime}$ as well as their fitting results using the Curie-Weiss law of $\epsilon^{\prime}$ $=C /\left(T-T_{0}\right)$, where $C$ and $T_{0}$ are the Curie constant and the Curie temperature, respectively. Two linear regions in the inverse dielectric constant has also been observed in SBN60 single crystals, which showed a crossover behavior at 630$680 \mathrm{~K} \cdot{ }^{37}$ It is interesting to note that this temperature is close to $T_{B}$.

$C_{11}$ and $C_{33}$ of KLN-a and KLN-b single crystals are shown in Fig. 6(a) and 6(b), respectively. For the calculation of the elastic constants, the reported refractive indices of KLN were used. ${ }^{38}$ The approximate ferroelectric phase tran-

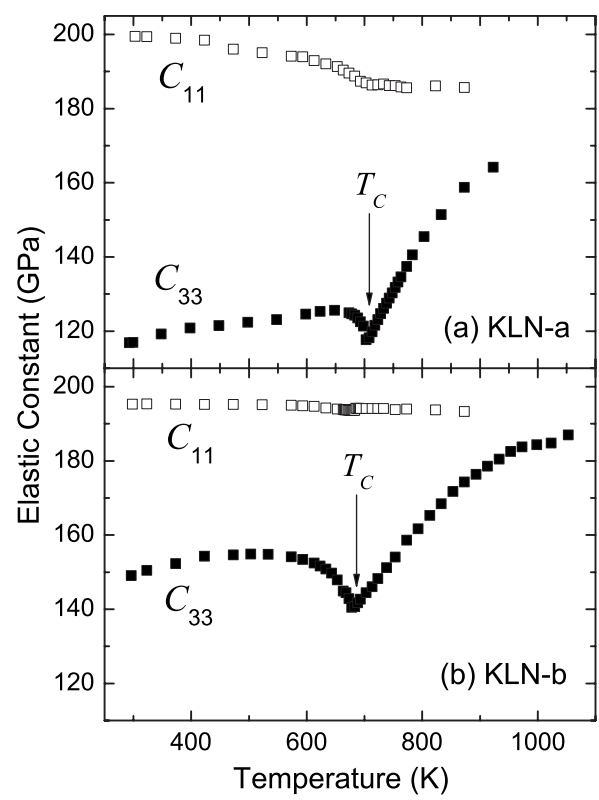

FIG. 6. The temperature dependences of $C_{11}$ and $C_{33}$ elastic constants of (a) KLN-a and (b) KLN-b single crystals. 
sition represented by the minimum temperature of $C_{33}$ is indicated by $T_{c}$. It is interesting to note that the observed $T_{c}$ by Brillouin scattering is lower than the dielectric maximum temperature $T_{m}$ detected by previous dielectric measurements $^{30,39}$ by about $20-30 \mathrm{~K}$. Since $T_{m}$ depends on the probe frequency due to the diffuse nature of the ferroelectric phase transition as well as to the conductivity contribution, the temperature determined by the minimum of $C_{33}$ should be considered as a true structural phase transition for these two KLN single crystals. Similar to SBN61 and KNS$\mathrm{BN}: \mathrm{Cu}$, only $C_{33}$ exhibits a significant softening upon approaching the ferroelectric phase transition from both highand low-temperature sides. $C_{11}$ shows a slight hardening in the low-temperature range below $T_{m}$. $C_{33}$ becomes hardened upon heating from $T_{m}$ and then seems to become saturated at very high temperatures.

\section{DISCUSSION}

\section{A. Acoustic anisotropy}

The common characteristic of the elastic properties observed from the four examined TB uniaxial ferroelectrics is that there is a large elastic anisotropy depending on the phonon propagation direction. $C_{33}$ shows a significant softening on approaching $T_{m}$ from both high- and low-temperature sides while $C_{11}$ does not display any substantial anomaly in the whole temperature range. Since the order parameter of these tetragonal TB ferroelectrics is a discrete onecomponent one of which the direction is along the fourfold polar $c$ axis, strong coupling between this Ising-type order parameter and the strain arising from the LA waves propagating along the same polar direction is expected. The fluctuating local polarizations affected by the strain field of the LA waves in turn respond to the elastic system resulting in a change in the elastic stiffness coefficient.

The $C_{33}$ of SBN61 becomes softened upon cooling via three steps occurring in three temperature ranges divided by two characteristic temperatures, $T_{B}$ and $T^{*}$. Above $T_{B}, C_{33}$ continuously decreases with lowering temperature, which is in contrast to the temperature dependence of $C_{33}\left(=C_{11}\right)$ of all investigated cubic relaxor perovskites. ${ }^{20-26}$ The possible origin of this anomalous behavior will be discussed in the following section. Near $T_{B}$, a slight change in the slope of $\nu_{B}$ can be noticed from Fig. 2 indicating some change in the nature of the relevant degree of freedom coupled to the acoustic waves, probably due to the formation of PNRs. The existence of $T_{B}$ in SBN has been indicated by many experimental results such as the measurements of the strain and the linear birefringence. ${ }^{11,12}$ In the temperature range between $T_{B}$ and $T^{*}$ becomes softened $C_{33}$ with almost temperature independent acoustic damping. Since there is no macroscopic spontaneous polarization in the paraelectric or ergodic relaxor phase between $T^{*}$ and $T_{B}$, we expect electrostrictive coupling would dominate the interaction between the PNRs and the strain. In this case, the critical part of $C_{33}$, i.e., the decrease in $C_{33}$ arising from the electrostrictive coupling can be expressed by $g_{33}^{2}\left\langle P_{3}^{2}\right\rangle \chi$, where $g_{33}$ is the relevant electrostrictive coefficient, $\left\langle P_{3}^{2}\right\rangle$ is the mean value of the squared local polarization of PNRs aligned in the direction along the $c$ axis, and $\chi$ is the susceptibility. ${ }^{40}$ Fluctuating local polarizations appear at $T_{B}$ and then gradually grow with decreasing temperature in RFEs. Although the direction of these polarizations are random, their appearance contributes to the decrease in the elastic constant owing to the quadratic nature of the electrostrictive interaction. This decrease would evolve continuously without substantial change because the growth of $\left\langle P_{3}^{2}\right\rangle$ is very gradual in a wide temperature range until the interaction between PNRs becomes stronger and thus the PNRs get long lived and more correlated than at higher temperatures. Indication to this change in the dynamics of PNRs can be noticed at $T^{*} \sim 450 \mathrm{~K}$ from the more steepened slope in $\nu_{B}$, sudden increase in the FWHM of the LA mode, the increase in the intensity of the second harmonic generation signal, ${ }^{33}$ as well as the integrated intensity of CPs. ${ }^{28} \nu_{B}$ and $C_{33}$ become minimum at the diffuse phase transition of $\sim 340 \mathrm{~K}$, concomitant with the maximized hypersonic damping as can be seen in Fig. 2. Significant increase in the FWHM appearing below $T^{*}$ suggests that the contribution to the hypersonic attenuation by the order parameter fluctuations becomes more substantial due to the stronger intercluster correlations than those between PNRs at higher temperatures.

Acoustic properties of KNSBN:Cu shown in Fig. 4 exhibit dynamical changes similar to those observed in SBN61. The addition of $\mathrm{K}$ and $\mathrm{Na}$ to $\mathrm{SBN}$ reduces the number of vacancies of KNSBN:Cu to half of that of SBN, which increases the Curie temperature and reduces the relaxor behaviors. KNSBN: $\mathrm{Cu}$ shows a definite structural phase transition at about $420 \mathrm{~K}$ from $4 / \mathrm{mmm}$ to $4 \mathrm{~mm}$ symmetry, but this transition is diffused to some degree. ${ }^{29,36}$ The dielectric maximum temperature depends on the probe frequency slightly, and the inverse dielectric constant shows a deviation from the Curie-Weiss law at temperatures close to $T_{m}$. It should be noted that the inverse dielectric constant exhibits two linear regions in the paraelectric phase divided by a crossover temperature of about $530 \mathrm{~K}$. Above this temperature, $\nu_{B}$ of the LA waves propagating along the $c$ axis decreases upon cooling with a temperature independent hypersonic damping factor. Below $530 \mathrm{~K}$, the FWHM begins to increase slightly accompanied by continuous decrease in $\nu_{B}$ with decreasing temperature. When the inverse dielectric constant deviates from the Curie-Weiss behavior at about $430 \mathrm{~K}, \nu_{B}$ becomes more steepened and the FWHM shows a sharp increase. $\nu_{B}$ attains a minimum at $T_{m}$. The high value of FWHM in the ferroelectric phase below $T_{m}$ seems to reflect the damping arising from the phonon scattering by ferroelectric domain walls. Regarding to $\mathrm{CP}$ behaviors of KNSBN:Cu, two-component CPs have been observed, and both showed substantial increase, i.e., growth of the integrated intensity below $\sim 500 \mathrm{~K}$ upon cooling. ${ }^{41}$

Two KLN single crystals show similar behaviors. Both KLN-a and KLN-b show a dip in $C_{33}$ at the phase transition temperature but no appreciable acoustic anomaly in $C_{11}$ except slight hardening below $T_{c}$. Although there has been no report about the existence of $T_{B}$ in these systems, several experimental evidences for the existence of precursor polar clusters above $T_{c}$ have been suggested for KLN single crystals, for example, appearance of a strongly polarized central 


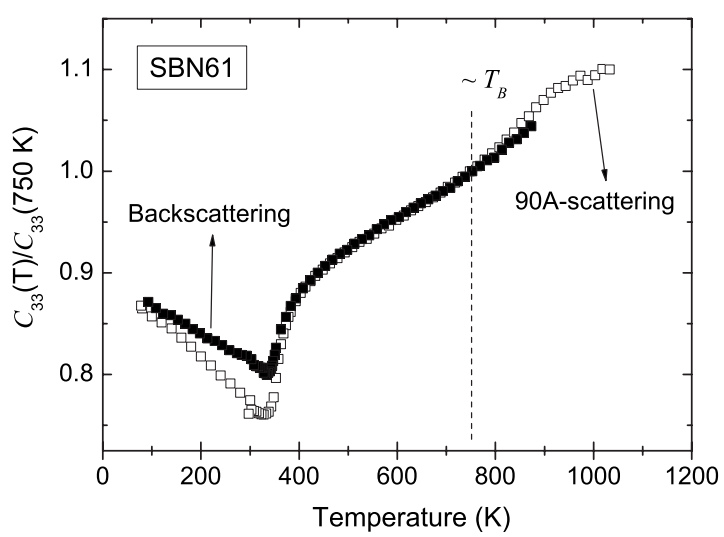

FIG. 7. The temperature dependence of the normalized elastic constant $C_{33}$ of SBN61 with respect to the value at $T=750 \mathrm{~K}$.

peak $^{39,42}$ and deviation of the dielectric constant from the Curie-Weiss law in the paraelectric phase. ${ }^{39}$ The appearance and the growth of CP of KLN-b are accompanied by a substantial increase in the hypersonic damping of the LA mode corresponding to $C_{33} \cdot{ }^{39,42}$ In addition, the relaxation time estimated from the width of CP shows a slowing-down behavior with decreasing temperature. All these results obtained from the four TB uniaxial single crystals suggest that the acoustic anisotropy between $C_{11}$ and $C_{33}$ arises from the coupling between the one-component order parameter of precursor polar clusters aligned along the polar $c$ axis and the strain field arising from the LA waves propagating along the same direction. Common characteristics to all samples are significant softening of $C_{33}$, substantial growth of hypersonic damping, appearance and growth of $\mathrm{CP}$, and slowing down of the relevant dynamics of $\mathrm{CP}$ represented by the reducing half width, all of which have been observed on approaching $T_{m}$ or $T_{c}$ from high-temperature side and are believed to be correlated with the evolution of precursor polar clusters.

\section{B. Acoustic dispersion in SBN61}

Two $C_{33}$ 's of SBN61 measured from two scattering geometries, which show slight differences at high temperatures, have been normalized to the value obtained at $750 \mathrm{~K}$ $\left(\sim T_{B}\right)$, and $C_{33}(T) / C_{33}(T=750 \mathrm{~K})$ is shown in Fig. 7 . The temperature dependences of the two normalized elastic constants are almost the same in the temperature range of 400 $870 \mathrm{~K}$. A dispersion starts to appear at about $380 \mathrm{~K}$, becomes the largest at $T_{m}$ and gradually disappears on lowering temperature. The minimum temperature of $C_{33}(T)$ depends on the wavevector of the LA waves slightly and is located at a higher temperature for the LA waves having a larger wavevector and thus a higher probe frequency at the backward scattering geometry. Similar acoustic dispersion has also been observed in the archetypical relaxor $\mathrm{Pb}\left(\mathrm{Mg}_{1 / 3} \mathrm{Nb}_{2 / 3}\right) \mathrm{O}_{3}$ (PMN) system. ${ }^{26}$ The onset of the acoustic dispersion in PMN was suggested to correlate with cubic anisotropy.

When PNRs form at and grow below $T_{B}$ in SBN61, they can move freely under the strain field by the acoustic waves and then respond to the acoustic waves, which can be described by a relaxation process. ${ }^{43}$ Since the intercluster inter- action becomes stronger between PNRs with decreasing temperature, the mean relaxation time for the response of the order parameter to the strain field is expected to shift to larger values and the distribution of the relaxation time would become broader upon cooling toward $T_{m}$. ${ }^{44}$ Broadband dielectric spectroscopic studies have revealed that the distribution function of the dielectric relaxation time in SBN also becomes broader with decreasing temperature. ${ }^{45,46}$ At high temperatures where the relaxation frequencies of the ordering quantity are much higher than the frequency of the LA waves, the order parameter can respond to the strain field instantaneously contributing to the softening of the related elastic constant and differentiating it from the infinitefrequency elastic constant. As the substantial portion of the distribution of the acoustic relaxation times becomes longer and comparable to the inverse of the frequency of the acoustic waves, many PNRs cannot respond to the elastic system and do not contribute to the softening of the elastic constant. The higher the LA mode frequency having larger wavevector, the higher the temperature at which this process would occur. Coarsening and stabilization of PNRs due to increased intercluster correlation observed at $360 \mathrm{~K}$ by dielectric study ${ }^{47}$ may affect the distribution of the acoustic relaxation times contributing to the onset of the acoustic dispersion at similar temperatures as shown in Fig. 7.

\section{Acoustic behavior of SBN61 above $T_{B}$}

The most noteworthy result of the present study is that $\nu_{B}$ becomes larger continuously with increasing temperature, even when crossing $T_{B}$ in the case of SBN61. $\nu_{B}$ 's tend to saturate at the highest measurement temperatures but do not show any maximum in the investigated temperature ranges. In the case of cubic perovskite relaxors, $\nu_{B}$ first increases and then decreases upon heating, and the maximum temperature for $\nu_{B}$ corresponds to $T_{B}$ approximately. ${ }^{20-26}$ Linear decreasing behavior of $\nu_{B}$ upon heating above $T_{B}$ is consistent with the theoretical prediction based on normal lattice anharmonicity. ${ }^{48}$ The continuous hardening of $C_{33}$ upon heating is quite an unexpected result because the hightemperature regions above $T_{B}$ is simply a paraelectric phase different from the ergodic relaxor phase below $T_{B}$.

It should be emphasized that, similar to $\mathrm{KNSBN}: \mathrm{Cu}$, the SBN61 also shows two linear regions in the inverse dielectric constant characterized by two different sets of Curie parameters, which are divided by a crossover temperature close to $T_{B}{ }^{37}$ This dielectric behavior is different from those of $\mathrm{Pb}$-based perovskite relaxors in which the dielectric constant shows deviation from the Curie-Weiss law at $T_{B}$. The acoustic properties of the LA waves propagating along the $c$ axis show different behaviors when the temperature changes across the crossover temperature which divides the inverse dielectric constant into two linear regions. $\nu_{B}$ displays a continuous decrease upon cooling while the FWHM, which is related to the hypersonic damping, is almost temperature independent in the low-temperature region, i.e., from $T_{B}$ to $T^{*}$. The change in the Curie parameters at the crossover temperature may reflect the change in the magnitude of dipole moments and the strength of the dipole couplings due to the 
formation of PNRs at $T_{B}$. Recently, broadband dielectric spectroscopy revealed that a single-particle relaxation persisting up to $900 \mathrm{~K}$ and not existing in $\mathrm{Pb}$-based perovskite relaxors changes into a collective relaxation of PNRs indicating a crossover behavior in the temperature evolution of the dynamics of SBN and Na-doped SBN. ${ }^{49}$ Based on these findings, continuation of the softening of $\nu_{B}$ and $C_{33}$ above $T_{B}$ in SBN61 observed during cooling, as can be seen from Figs. 2 and 3, may tentatively be ascribed to the coupling between the single-particle relaxators inherent only in unfilled TB uniaxial crystals and the LA waves. Further gradual decrease in FWHM of the LA waves upon heating above $T_{B}$ may hint at the existence of additional relaxation process which can couple to the acoustic waves. It would be very interesting to investigate whether the high-frequency sinigleparticle relaxation, observed from SBN, exists in other unfilled TB ferroelectrics such as KNSBN:Cu and KLN single crystals. Finally, it should be mentioned that in addition to the single-particle relaxation other effects such as the increase in oxygen vacancies and ionic conductivity might be related to the acoustic behaviors above $T_{B}$, which needs additional investigation.

\section{CONCLUSION}

We investigated acoustic properties of four uniaxial tungsten bronze single crystals, $\left(\mathrm{Sr}_{0.61} \mathrm{Ba}_{0.39}\right)_{5} \mathrm{Nb}_{10} \mathrm{O}_{30}$, $\left(\mathrm{K}_{0.5} \mathrm{Na}_{0.5}\right)_{1.0}\left(\mathrm{Sr}_{0.75} \mathrm{Ba}_{0.25}\right)_{4.5} \mathrm{Nb}_{10} \mathrm{O}_{30}, \quad \mathrm{~K}_{5.80} \mathrm{Li}_{3.82} \mathrm{Nb}_{10.12} \mathrm{O}_{30}$, and $\mathrm{K}_{4.74} \mathrm{Li}_{3.07} \mathrm{Nb}_{10.44} \mathrm{O}_{30}$. Large acoustic anisotropy between $C_{33}$ and $C_{11}$ has been observed from all the investigated TB crystals, which has been ascribed to the existence of the Ising-type one-component order parameter of the precursor polar clusters and its electrostrictive coupling to the strain field of the acoustic waves. $C_{33}$ exhibits a substantial softening on approaching the diffuse phase transition from hightemperature side while $C_{11}$ does not show any significant anomaly. The softening of $C_{33}$ is accompanied by the increase in the hypersonic damping of the corresponding longitudinal acoustic waves, the appearance and the growth of the quasielastic central peaks, and the onset of acoustic dispersion within the Brillouin frequency window. The inverse dielectric constant of both SBN61 and KNSBN:Cu displays two linear regions having two different sets of Curie parameters divided by a crossover temperature being close to $T_{B}$ in the case of SBN61, which may indicate the change in the magnitude of dipole moments and the strength of the dipole couplings due to the formation of PNRs. $C_{33}$ of SBN61 shows continuous hardening upon heating even about $T_{B}$ along with decreasing hypersonic damping, which is distinguished from $\mathrm{Pb}$-based perovskite relaxors. This result may suggest the existence of additional relaxation process other than that of PNRs persisting at high temperatures above $T_{B}$ which couples to the acoustic waves. Recent observation of a single-particle relaxation at high temperatures and its transformation into a collective relaxation of PNRs by dielectric spectroscopy ${ }^{49}$ seems to be closely correlated with the anomalous acoustic behaviors which we observed in SBN61.

\section{ACKNOWLEDGMENTS}

This work was supported in part by the Korea Research Foundation Grant funded by the Korean Government (MOEHRD, Basic Research Promotion Fund) (Grant No. KRF2006-331-C00088).

${ }^{1}$ L. E. Cross, Ferroelectrics 76, 241 (1987).

${ }^{2}$ G. Burns and F. H. Dacol, Ferroelectrics 104, 25 (1990).

${ }^{3}$ A. A. Bokov and Z.-G. Ye, J. Mater. Sci. 41, 31 (2006).

${ }^{4}$ D. Viehland, S. J. Jang, L. E. Cross, and M. Wuttig, J. Appl. Phys. 68, 2916 (1990).

${ }^{5}$ N. de Mathan, E. Husson, G. Calvarin, J. R. Gavarri, A. W. Hewat, and A. Morell, J. Phys.: Condens. Matter 3, 8159 (1991).

${ }^{6}$ G. Burns and F. H. Dacol, Solid State Commun. 48, 853 (1983).

${ }^{7}$ R. Blinc, V. V. Laguta, B. Zalar, and J. Banys, J. Mater. Sci. 41, 27 (2006).

${ }^{8}$ W. Kleemann, J. Mater. Sci. 41, 129 (2006).

${ }^{9}$ B. Chaabane, J. Kreisel, B. Dkhil, P. Bouvier, and M. Mezouar, Phys. Rev. Lett. 90, 257601 (2003).

${ }^{10}$ K. Hirota, Z.-G. Ye, S. Wakimoto, P. M. Gehring, and G. Shirane, Phys. Rev. B 65, 104105 (2002).

${ }^{11}$ A. S. Bhalla, R. Guo, L. E. Cross, G. Burns, F. H. Dacol, and R. R. Neurgaonkar, Phys. Rev. B 36, 2030 (1987).

${ }^{12}$ P. Lehnen, W. Kleemann, Th. Woike, and R. Pankrath, Eur. Phys. J. B 14, 633 (2000).

${ }^{13}$ E. Dul'kin, M. Roth, P.-E. Janolin, and B. Dkhil, Phys. Rev. B 73, 012102 (2006).

${ }^{14}$ S. Tinte, B. P. Burton, E. Cockayne, and U. V. Waghmare, Phys. Rev. Lett. 97, 137601 (2006)

${ }^{15}$ J.-H. Ko, S. Kojima, A. A. Bokov, and Z.-G. Ye, Appl. Phys. Lett. 91, 252909 (2007).

${ }^{16}$ P. B. Jamieson, S. C. Abrahams, and J. L. Bernstein, J. Chem. Phys. 48, 5048 (1968).

${ }^{17}$ R. Pirc and R. Blinc, Phys. Rev. B 60, 13470 (1999).

${ }^{18}$ W. Kleemann, J. Dec, V. V. Shvartsman, Z. Kutnjak, and T. Braun, Phys. Rev. Lett. 97, 065702 (2006).

${ }^{19}$ S. D. Prokhorova and S. G. Lushnikov, Ferroelectrics 90, 187 (1989).

${ }^{20}$ J.-H. Ko, S. Kojima, and S. G. Lushnikov, Appl. Phys. Lett. 82, 4128 (2003).

${ }^{21}$ G. Shabbir, J.-H. Ko, S. Kojima, and Q.-R. Yin, Appl. Phys. Lett. 82, 4696 (2003).

${ }^{22}$ S. G. Lushnikov, J.-H. Ko, and S. Kojima, Appl. Phys. Lett. 84, 4798 (2004).

${ }^{23}$ J.-H. Ko, D. H. Kim, S. Kojima, W. Chen, and Z.-G. Ye, J. Appl. Phys. 100, 066106 (2006).

${ }^{24}$ S. Tsukada, Y. Ike, J. Kano, T. Sekiya, Y. Shimojo, R. Wang, and S. Kojima, Appl. Phys. Lett. 89, 212903 (2006).

${ }^{25}$ J.-H. Ko, D. H. Kim, and S. Kojima, Phys. Rev. B 77, 104110 (2008).

${ }^{26}$ S. G. Lushnikov, A. I. Fedoseev, S. N. Gvasaliya, and S. Kojima, Phys. Rev. B 77, 104122 (2008).

${ }^{27}$ F. M. Jiang, J.-H. Ko, and S. Kojima, Phys. Rev. B 66, 184301 (2002).

${ }^{28}$ J.-H. Ko and S. Kojima, Appl. Phys. Lett. 91, 082903 (2007).

${ }^{29}$ I. G. Siny, S. G. Lushnikov, S. I. Siny, V. H. Schmidt, A. A. Savvinov, and R. S. Katiyar, J. Appl. Phys. 89, 1671 (2001).

${ }^{30}$ J.-H. Ko, S. Kojima, S. G. Lushnikov, R. S. Katiyar, T.-H. Kim, and J.-H. Ro, J. Appl. Phys. 92, 1536 (2002).

${ }^{31}$ Y. Gorouya, Y. Tsujimi, Y. Yamashita, T. Ifukube, and T. Yagi, Ferroelectrics 266, 165 (2002).

${ }^{32}$ Th. Woike, T. Granzow, U. Dorfler, Ch. Poetsch, M. Wohlecke, and R. Pankrath, Phys. Status Solidi A 186, R13 (2001).

${ }^{33}$ P. Lehnen, J. Dec, W. Kleemann, Th. Woike, and R. Pankrath, Ferroelectrics 240, 1547 (2000).

${ }^{34}$ J. M. Vaughan, The Fabry-Pérot Interferometer (The Adam Hilger, Bristol, 1989), p. 348.

${ }^{35}$ S. C. Abrahams, P. B. Jamieson, and J. L. Bernstein, J. Chem. Phys. 54, 2355 (1971)

${ }^{36}$ J.-H. Ko, D. H. Kim, S. G. Lushnikov, R. S. Katiyar, and S. Kojima, Ferroelectrics 286, 61 (2003).

${ }^{37}$ W. H. Huang, D. Viehland, and R. R. Neurgaonkar, J. Appl. Phys. 76, 490 (1994).

${ }^{38}$ T. Karaki, K. Miyashita, M. Nakatsuji, and M. Adachi, Jpn. J. Appl. Phys. 
37, 5277 (1998).

${ }^{39}$ J.-H. Ko, B.-E. Jun, Y. H. Hwang, D. H. Kim, and S. Kojima, J. Korean Phys. Soc. (in press).

${ }^{40}$ G. A. Smolenskii, N. K. Yushin, and S. I. Smirnov, Sov. Phys. Solid State 27, 492 (1985).

${ }^{41}$ S. G. Lushnikov (unpublished).

${ }^{42}$ J.-H. Ko, D. H. Kim, and S. Kojima, J. Korean Phys. Soc. 51, S67 (2007).

${ }^{43}$ W. Rehwald, Adv. Phys. 22, 721 (1973).

${ }^{44}$ S. N. Dorogovtsev and N. K. Yushin, Ferroelectrics 112, 27 (1990).
${ }^{45}$ E. Buixaderas, M. Savinov, M. Kempa, S. Veljko, S. Kamba, J. Petzelt, R. Pankrath, and S. Kapphan, J. Phys.: Condens. Matter 17, 653 (2005).

${ }^{46}$ J. Banys, J. Macutkevic, R. Grigalaitis, and W. Kleemann, Phys. Rev. B 72, 024106 (2005).

${ }^{47}$ J. Dec, V. V. Shvartsman, and W. Kleemann, Appl. Phys. Lett. 89, 212901 (2006).

${ }^{48}$ T. Sonehara, E. Tatsu, and S. Saikan, J. Appl. Phys. 101, 103507 (2007).

${ }^{49}$ A. Belous, O. V'yunov, D. Mishchuk, S. Kamba, and D. Nuzhnyy, J. Appl. Phys. 102, 014111 (2007) 